\title{
Actinomicosis diseminada en un paciente inmunocompetente: Reporte de caso
}

\author{
Erick Sandoval-Mallma*1,a; José Caballero-Silva 1,b
}

RESUMEN

Se reporta el caso de un paciente de 58 años, quien acude al hospital con tiempo de enfermedad de 8 meses caracterizado por múltiples abscesos en piel y tejidos blandos, asociado a pérdida de peso de aproximadamente $20 \mathrm{~kg}$, hipoalbuminemia severa, anemia moderada y poliserositis. Se realizaron diversos estudios de imágenes que evidenciaron colecciones en plano muscular de muslo izquierdo. Además, en la ecografía de abdomen, pelvis y tórax se reportó nefropatía intersticial bilateral, ascitis y derrame pleural bilateral. El estudio histopatológico confirmó el diagnóstico de actinomicosis. Por las manifestaciones sistémicas y la distribución difusa de los abscesos, clínicamente correspondía a un caso de actinomicosis diseminada, por lo que recibió tratamiento antibiótico endovenoso con penicilina G sódica por 4 semanas, y luego, amoxicilina por vía oral. El paciente evolucionó favorablemente y los abscesos remitieron.

Palabras clave: Actinomicosis; Supuración; Piel (Fuente: DeCS BIREME).

\section{Disseminated actinomycosis in an immunocompetent patient: a case report}

\section{ABSTRACT}

This case report describes a 58-year-old patient who visited the hospital after an 8-month disease period characterized by multiple skin and soft tissue abscesses associated with an approximately 20-kg weight loss, severe hypoalbuminemia, moderate anemia and polyserositis. Several imaging tests revealed abscesses on the left thigh muscle. Additionally, abdomen, pelvis and thorax ultrasounds showed bilateral interstitial nephropathy, ascites and bilateral pleural effusion. The histopathological examination confirmed the diagnosis of actinomycosis. Given the systemic manifestations and diffuse distribution of the abscesses, the case was clinically consistent with disseminated actinomycosis. Therefore, the patient received IV antibiotic treatment with penicillin $\mathrm{G}$ sodium for 4 weeks and then with amoxicillin p.o. The patient evolved favorably and exhibited abscess remission.

Keywords: Actinomycosis; Suppuration; Skin (Source: MeSH NLM).

1. Hospital Nacional Arzobispo Loayza. Lima, Perú.

a. Médico Infectólogo y Tropicalista.

b. Médico Internista.

*Autor corresponsal 


\section{INTRODUCCIÓN}

La actinomicosis es una infección endógena poco frecuente de curso subagudo a crónico. Es causada por bacterias filamentosas Gram positivas, anaerobias o microaerófilas pertenecientes, principalmente, al género Actinomyces spp. (la más frecuente es A.israelii). Estas bacterias forman parte de la flora normal de la cavidad oral, tracto gastrointestinal y tracto genital femenino y, generalmente, son poco virulentas; sin embargo, cuando hay disrupción de la membrana mucosa pueden invadir los tejidos y generar lesiones supurativas granulomatosas con fistulización a las áreas adyacentes; además, pueden diseminarse por vía hematógena o linfática. La enfermedad afecta la región facial-cervical en aproximadamente el $50 \%$ de los casos, el tórax en 20$30 \%$, el abdomen en alrededor de $10-20 \%$, y la región pélvica en cerca al 3-5\%. Rara vez la diseminación hematógena puede llegar a comprometer varios órganos, y originar múltiples lesiones nodulares, que simulan el comportamiento de tumores malignos metastásicos ${ }^{(1-5)}$.

La actinomicosis es una enfermedad poco frecuente, su distribución es universal, pero es más habitual en regiones o grupos con situación socioeconómica o sistemas de salud deficientes. Este cuadro es diez veces mayor en las comunidades rurales que en las áreas urbanas, parece afectar tres veces más a hombres que a mujeres; y es más frecuente entre los 20 a 60 años de edad. Los factores de riesgo que se asocian a esta enfermedad son la presencia de dispositivos protésicos (por ejemplo, dispositivos intrauterinos), alcoholismo e inadecuada higiene dental. La mortalidad de la actinomicosis oscila entre 0 a $28 \%$, y está influenciada, principalmente, por un estado de inmunosupresión (por ejemplo, diabetes, infección por $\mathrm{VIH}$, entre otros), además del tiempo y la extensión de la enfermedad ${ }^{(1-3)}$.

Para establecer el diagnóstico de actinomicosis contamos con diferentes exámenes, como la tinción Gram, el cultivo en medio anaerobio con $\mathrm{CO}_{2}$ de 4 a $10 \%$, la identificación de los gránulos de azufre con histopatología (que pueden encontrarse en otros cuadros como nocardiosis y eumicetomas) y biología molecular (PCR). Sin embargo, a pesar de contar con varios medios de aislamiento, en ocasiones es difícil identificar al microorganismo y se diagnostica en base a indicios clínicos y laboratoriales (generalmente, tinción Gram positiva o presencia de gránulos de azufre, o ambos) e inclusive, en algunos casos, a través de la prueba terapéutica ${ }^{(2,4)}$.

El tratamiento de la actinomicosis es prolongado; se inicia con penicilina $\mathrm{G}$ en dosis altas (de 12 a 24 millones de $\mathrm{Ul}$ por día) por vía parenteral por 2 a 6 semanas, seguida por un régimen por vía oral, hasta completar un curso de 6 a 12 meses de tratamiento. Si el paciente es alérgico a penicilinas, pueden utilizarse tetraciclina, macrólidos o lincomicinas (clindamicina), entre otros ${ }^{(1-3)}$. Algunos autores sugieren acortar la duración del tratamiento hasta 2 meses, y agregar metronidazol al esquema inicial para cubrir otras bacterias anaerobias que potencian el crecimiento del actinomices, sin embargo, esta práctica es cuestionable y solo se aplica a casos de actinomicosis cérvico-facial leve.

La mayoría de países de América Latina no cuenta con datos de prevalencia de actinomicosis en la población. Solo en Colombia, se han reportado 400 casos anuales entre los años 2009 y 2013, según su sistema de información SISPROCubo de Datos ${ }^{(6)}$. En el Perú, se han reportado casos de actinomicosis regional, pero no se ha estimado el impacto de enfermedad en nuestra población; además, no existen reportes recientes de compromiso diseminado ${ }^{(7)}$.

Por tal motivo, presentamos el caso de un paciente varón de 58 años, diagnosticado de actinomicosis cutánea diseminada mediante histopatología y que recibió tratamiento antibiótico con penicilina $G$ sódica por vía endovenosa al inicio y luego amoxicilina por vía oral, y tuvo una evolución favorable.

\section{CASO CLÍNICO}

Paciente varón de 58 años, natural y procedente de Lima, Perú, que trabaja como estadístico, con antecedentes de consumo de tabaco y alcohol de forma esporádica. Un hermano con diagnóstico de cáncer gástrico en tratamiento con quimioterapia. Sin otros antecedentes significativos.

Ingresó a la institución por emergencia con un tiempo de enfermedad de 8 meses. El cuadro inicia con lesiones tumorales induradas en miembros inferiores y superiores; 30 días antes del ingreso las lesiones aumentan de tamaño y se agrega dolor de baja intensidad, asociado a supuración de una de las tumoraciones a nivel inguinal; además, se presentan fiebre, postración y pérdida de peso de aproximadamente 20 kilogramos.

Al examen físico inicial, presentaba presión arterial de $100 / 60 \mathrm{mmHg}$, frecuencia cardiaca de 86 latidos por min, frecuencia respiratoria de 18 respiraciones por min, saturación de $\mathrm{O}_{2}$ de $98 \%$ y temperatura de $39{ }^{\circ} \mathrm{C}$. Se encontraba en regular estado general, con fascia doloroso, orientado en tiempo, espacio y persona, palidez de piel y mucosas, y edemas en ambos miembros inferiores. A nivel de piel y tejidos blandos presentaba múltiples lesiones fluctuantes, supurativas, sin signos de flogosis ("abscesos 
fríos") en brazo derecho, muslo izquierdo, en abdomen a nivel de los flancos (Figura 1) y una úlcera supurativa en el área inguinal derecha (Figura 2A). En la cavidad oral se evidenciaron lesiones ulcerativas adyacentes a la primera y tercera molar superior derecha (Figura 2B). Las evaluaciones cardiorrespiratoria, abdominal, genitourinaria y neurológica no presentaron hallazgos significativos.
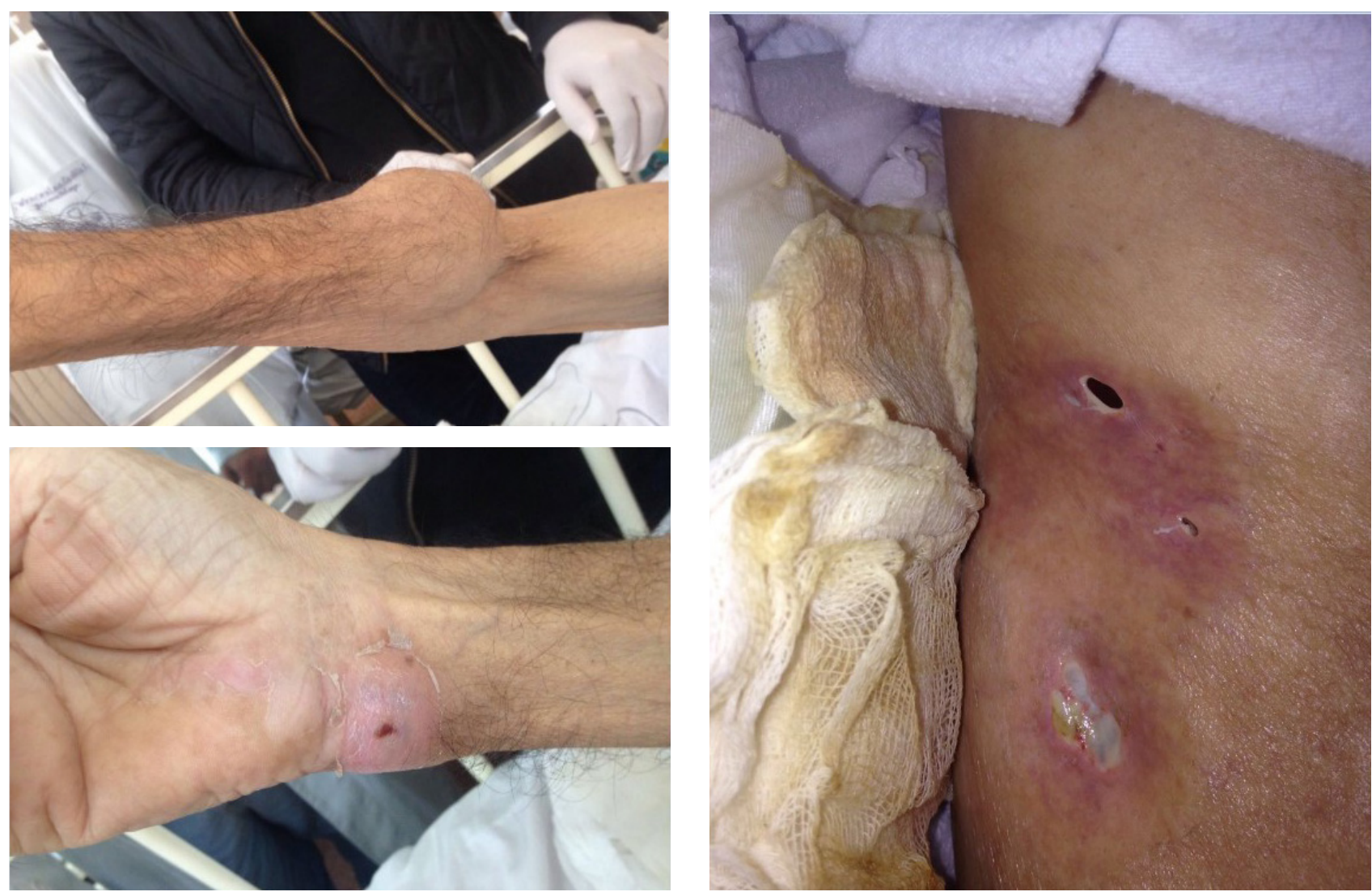

Figura 1. Abscesos cutáneos en codo derecho cara anterior, muñeca derecha y hemiabdomen derecho
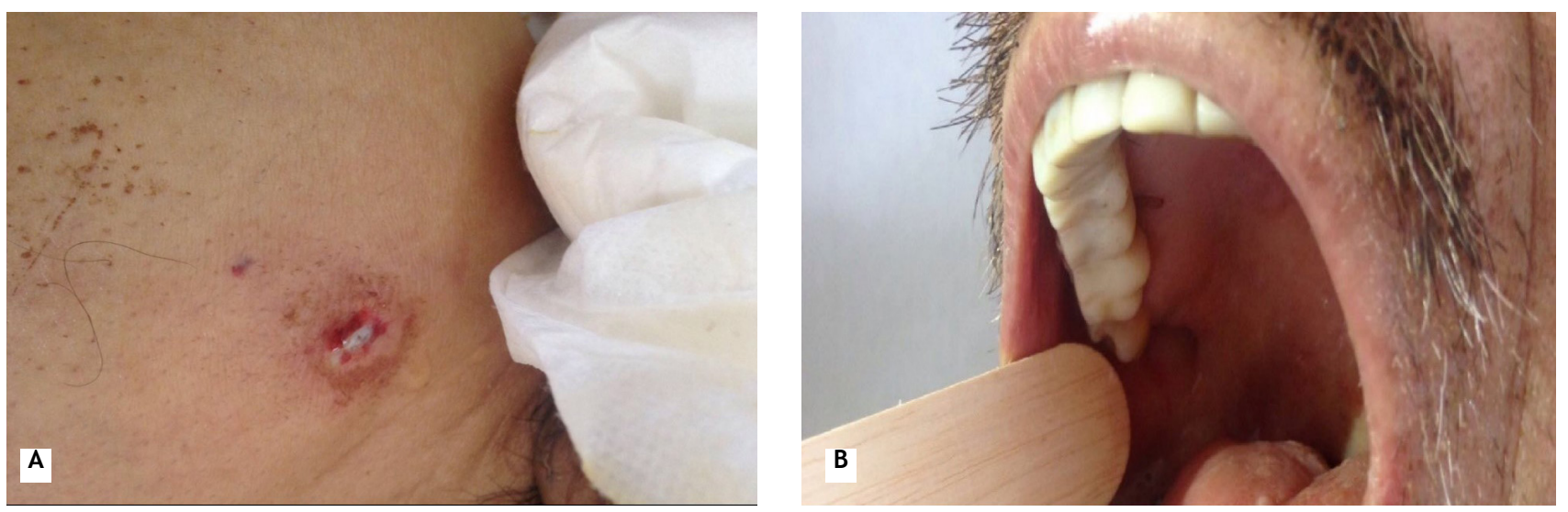

Figura 2. (A) Úlcera supurativa en región inguinal derecha, (B) Cavidad oral 
Tras la evaluación clínica se plantearon los diagnósticos diferenciales de piomiositis, micetoma, sífilis gomosa, actinomicosis y micosis subcutánea. Por tal motivo, se le solicitaron una serie de exámenes iniciales, cuyos resultados se detallan en la tabla 1 . En estos exámenes de ingreso se observaron una anemia moderada, asociada a leucocitosis con desviación izquierda, incremento de reactantes de fase aguda (proteína $C$ reactiva), e hipoalbuminemia severa. Los exámenes para evaluar la función hepática, perfil lipídico y examen de orina fueron normales. La serología para hepatitis B y C, VIH, HTLV-1, sífilis y marcadores tumorales fueron negativos.

Tabla 1. Resultados de exámenes de laboratorio al ingreso

\begin{tabular}{|c|c|c|}
\hline $\begin{array}{l}\text { Exámenes de } \\
\text { laboratorio }\end{array}$ & Resultados & Rangos de referencia \\
\hline Hemoglobina & 7,2 & 13 a $16 \mathrm{~g} / \mathrm{dl}$ \\
\hline Leucocitos & 13590 & 4500 y 10000 células/mcl \\
\hline Abastonados & $4 \%$ & $0-5 \%$ \\
\hline Segmentados & $88 \%$ & $40-85 \%$ \\
\hline Eosinófilos & 0 & $1-4 \%$ \\
\hline Basófilos & 0 & $0-4 \%$ \\
\hline Monocitos & $2 \%$ & $3-10 \%$ \\
\hline Linfocitos & $6 \%$ & $18-45 \%$ \\
\hline Plaquetas & 292000 & 150000 a $400000 \mathrm{u} / \mathrm{mcl}$ \\
\hline Sodio & 134 & $136-146 \mathrm{meq} / \mathrm{L}$ \\
\hline Potasio & 4,5 & $3,5-5,0 \mathrm{meq} / \mathrm{L}$ \\
\hline Cloro & 99 & $102-109 \mathrm{meq} / \mathrm{L}$ \\
\hline Proteínas totales & 5,4 & $6,7-8,6 \mathrm{~g} / \mathrm{dL}$ \\
\hline Albúmina & 1,8 & $3,5-5,5 \mathrm{~g} / \mathrm{dL}$ \\
\hline Globulina & 3,6 & $2,0-3,5 \mathrm{~g} / \mathrm{dL}$ \\
\hline TGO/AST & 20 & $12-38 \mathrm{U} / \mathrm{L}$ \\
\hline TGP/ALT & 20 & $7-41 \mathrm{U} / \mathrm{L}$ \\
\hline Glucosa & 128 & $82-110 \mathrm{mg} / \mathrm{dl}$ \\
\hline Urea & 33 & $18-55 \mathrm{mg} / \mathrm{dl}$ \\
\hline Creatinina & 0,56 & $0,6-1,2 \mathrm{mg} / \mathrm{dl}$ \\
\hline Proteína $\mathrm{C}$ reactiva & 5,5 & Hasta $1 \mathrm{mg} / \mathrm{L}$ \\
\hline
\end{tabular}

En la ecografía de partes blandas en muslo izquierdo, se observó colección en el plano muscular (Figura 3). En la ecografía de abdomen y pelvis se reportó nefropatía intersticial bilateral, ascitis, derrame pleural bilateral, engrosamiento de pared vesical e hipertrofia prostática. La radiografía de tórax y tomografía tóraco-abdominal evidenciaron derrame pleural bilateral (Figura 3). Se solicitaron los primeros cultivos de la secreción de abscesos, que fueron negativos; mientras que un hemocultivo resultó positivo para estafilococos coagulasa negativo. Luego se repitieron los cultivos de secreción de absceso que fueron positivos para Acinetobacter spp. Con estos resultados, el paciente recibió tratamiento antibiótico con meropenem y vancomicina, sin respuesta clínica favorable. 


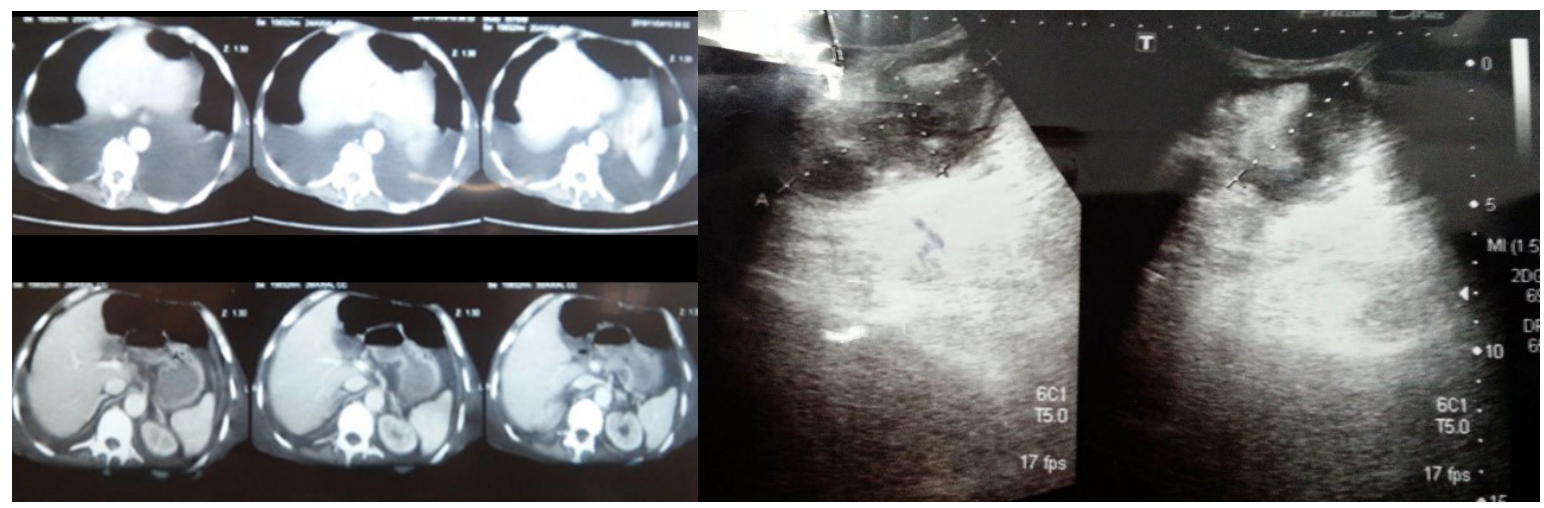

Figura 3. Tomografía toraco-abdominal y ecografía de piel y partes blandas

Durante la hospitalización se realizaron dos limpiezas quirúrgicas de las colecciones. Luego de 6 semanas de internamiento se obtuvo el resultado de la anatomía patológica de la biopsia de piel y músculo que mostró tejido fibroadiposo vascularizado con tejido de granulación y actinomicosis (gránulos basófilos de aspecto homogéneo y presencia de bacilos filamentosos Gram positivos) (Figura 4). Se inició tratamiento con penicilina $\mathrm{G}$ sódica (5 millones de UI) cada 6 horas por 28 días. Se observa remisión de las lesiones tumorales y de la fiebre al día 14 día de tratamiento. Luego de culminar la terapia por vía parenteral, se cambió a amoxicilina $500 \mathrm{mg}$ vía oral cada 8 horas. El cuadro evolucionó favorablemente, con la remisión total de las lesiones.

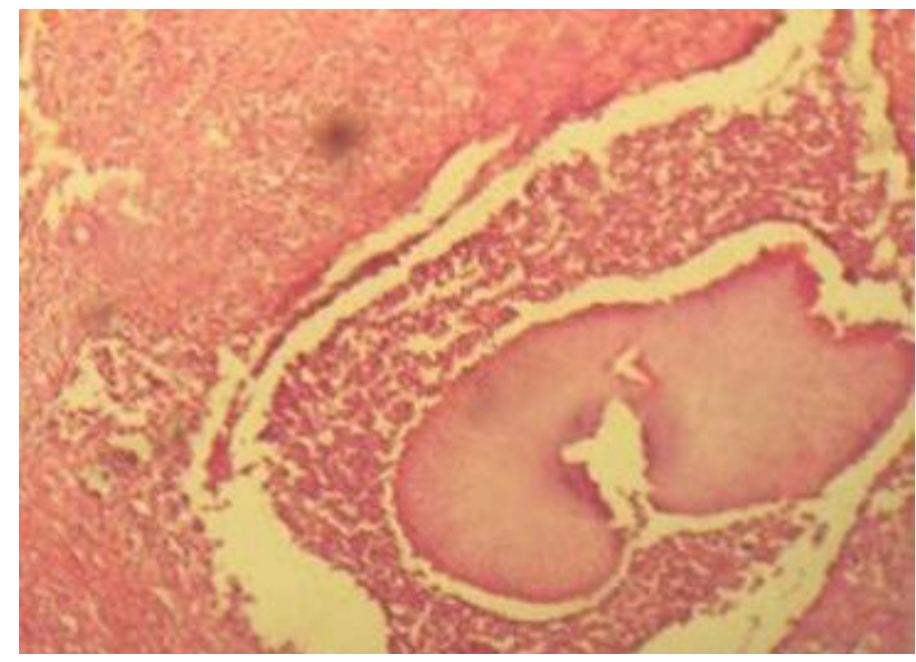

Figura 4. Resultado de anatomía patológica (gránulos de azufre)

\section{DISCUSIÓN}

El presente caso es relevante por lo infrecuente de la enfermedad y por lo raro de la presentación clínica. La actinomicosis puede afectar a muchos órganos y tejidos en el humano, y las manifestaciones pueden ser diversas y simular diferentes enfermedades como, por ejemplo, tumores localizados o metastásicos, tuberculosis, nocardiosis, infecciones fúngicas invasoras, sífilis tardía, entre otras. La frecuencia del cuadro varía según el lugar de la infección; así, el 50 a $65 \%$ de los casos aparecen en el área cérvicofacial; 15 a 30 \% se localizan en el tórax, 20 \% en la región abdomino-pélvica; 2 a 3 \% de los casos aparecen en el sistema nervioso central, mientras que las presentaciones musculoesqueléticas y diseminadas son raras ${ }^{(5)}$. El caso de nuestro paciente se encuentra en estos últimos dos grupos. 
La actinomicosis diseminada con compromiso de piel y tejidos blandos es una presentación clínica rara. Uno de los primeros casos fue descrito por Mounsey en 1947, que presentó el caso de un hombre de 43 años, con 2 años de enfermedad, quien, al ingreso al hospital, presentaba un síndrome de vena cava superior (circulación colateral toraco-braquial, edema en esclavina y cianosis facial), con lesiones ulcerativas supurativas en miembros inferiores y superiores; la evolución del paciente fue desfavorable y falleció. En la necropsia se identificó un compromiso extenso de ambos pulmones, asociado a una mediastinitis granulomatosa con infiltración de vasos sanguíneos, y se aisló Actynomices spp ${ }^{(8)}$.

Como se ha mencionado, los casos de actinomicosis diseminada son raros $y$, generalmente, tienen como foco primario el compromiso pulmonar. Si bien el compromiso torácico representa entre el $15-30 \%$ del total de casos de actinomicosis, la diseminación hematógena del Actinomices spp., incluso en estos casos, es rara; como lo demostraron Bates y Cruickshank, quienes, en una revisión de 85 casos de actinomicosis pulmonar, encontraron solo 2 de diseminación hematógena ${ }^{(9)}$. En el caso de nuestro paciente, no hubo compromiso pulmonar por parte de la actinomicosis, solo se halló un derrame pleural bilateral relacionado a la hipoalbuminemia severa.

Por otro lado, la actinomicosis diseminada de piel y tejidos blandos es más rara. Además del reporte de Mounsey, Savidge y Davies; en 1953, presentaron el caso de un joven de 18 años, con tiempo de enfermedad de 8 meses, caracterizado por malestar general, pérdida de peso progresiva, múltiples lesiones nodulares fluctuantes en brazos y piernas, un soplo sistólico en foco aórtico, asociado a compromiso pulmonar derecho. En el cultivo de la secreción de las lesiones cutáneas aislaron Actinomyces bovis, luego instauraron tratamiento antibiótico con penicilina por 2 meses con resolución de sus síntomas ${ }^{(8,10)}$. En 1970, Butas et al. reportaron un caso de actinomicosis diseminada con compromiso cutáneo a predominio de manos y pies, asociado a lesiones nodulares pulmonares, del cultivo de las lesiones cutáneas aislaron Actinomyces actinomicetemcomitans y Haemophilus aphrophilus, iniciaron tratamiento antibiótico con estreptomicina y tetraciclina por vía endovenosa, luego continuaron con penicilina hasta completar 3 meses de tratamiento, con evolución favorable y resolución de las lesiones ${ }^{(9)}$. Otro caso descrito por Smith et al. en la revista Chest, presenta el caso de un hombre de 39 años con antecedente de alcoholismo y tabaquismo, que fue hospitalizado por un cuadro respiratorio de dos meses de evolución asociado a lesiones supurativas en miembros inferiores y abdomen; en los estudios de anatomía patológica de las lesiones se evidenciaron gránulos de azufre y en la tinción Gram de las secreciones de las lesiones, estructuras filamentosas G (+), tras lo cual recibió tratamiento con penicilina $G$ potásica por 5 meses con resolución del cuadro ${ }^{(11)}$. Como se puede apreciar en los casos descritos, la actinomicosis diseminada cutánea suele acompañarse de compromiso visceral; principalmente, a nivel pulmonar; sin embargo, en nuestro paciente el compromiso es predominantemente de partes blandas.

Un aspecto que merece mención en el presente caso es la falta de factores de riesgos o inmunosupresión que expliquen el compromiso extenso de la actinomicosis. En diversos reportes se observa una asociación entre el alcoholismo y la actinomicosis; pero nuestro paciente no contaba con ese antecedente, ya que según la definición de la OMS, su consumo del alcohol no correspondía a la de un alcohólico. Por otro lado, sí presentaba pobre higiene dental y lesiones en cavidad oral, que pudieron predisponer el inicio del cuadro. Al ampliar el estudio para descartar condiciones inmunosupresoras, los exámenes de laboratorio resultaron negativos para VIH, HTLV-1 y marcadores tumorales; además, en la tomografía toracoabdominal no se evidenció la presencia de lesiones tumorales ${ }^{(1-3,12)}$. Existen algunos reportes de actinomicosis diseminada con compromiso primariamente cutáneo en pacientes inmunosuprimidos; uno de ellos es el de Takeda et al. quienes reportaron el caso de una paciente de 42 años con leucemia linfocítica aguda que desarrolló un cuadro de actinomicosis con diseminación principalmente en piel; otros reportes presentan como factores de riesgo de actinomicosis diseminada, la presencia de neoplasias, diabetes mellitus, uso de corticoides, entre otros ${ }^{(12)}$.

Con respecto al método de diagnóstico, es conocido que entre el 5,5 - $50 \%$ de los cultivos para Actynomices spp., pueden resultar negativos, y que de los casos con cultivos negativos el $31 \%$ pueden ser diagnosticados gracias a los estudios histopatológicos, en los que se pueden evidenciar los "gránulos de azufre" que muestran una parte central basófila y un borde radiado eosinófilo por tinción hematoxilina-eosina y la presencia de bacilos Gram positivos; este último hallazgo es compatible con nuestro paciente ${ }^{(1-3)}$. Existen otros métodos diagnósticos como, por ejemplo, la secuenciación del gen $16 \mathrm{~S}$ rRNA, la hibridación ADN-ADN, PCR en tiempo real y la hibridación in situ con fluorescencia. Sin embargo, a pesar de contar con varios métodos, el diagnóstico definitivo puede ser complicado $y$ en ocasiones puede realizarse la prueba terapéutica en cuadros clínicos compatibles con actinomicosis. Otro aspecto importante, en el presente caso, fue el aislamiento de estafilococos coagulasa negativo y Acinetobacter spp., los cuales, según la evolución clínica del paciente, corresponden a contaminaciones de la muestra; sin embargo, no debemos olvidar que puede existir coinfección en algunos casos de actinomicosis, que potencian el crecimiento de esta bacteria ${ }^{(12)}$.

Como se ha mencionado, en muchos casos el tratamiento 
antibiótico con penicilina es suficiente; sin embargo, en algunas ocasiones es necesario el desbridamiento o drenaje quirúrgico de las lesiones, sobre todo en las presentaciones torácicas. En el caso de nuestro paciente, los drenajes y las limpiezas quirúrgicas se realizaron antes de conocer el diagnóstico, pero una vez instalado el tratamiento antibiótico dirigido (penicilina G sódica), no hubo necesidad de otro procedimiento quirúrgico. La mayoría de casos de actinomicosis evolucionan favorablemente una vez que se inicia el tratamiento antibiótico, como sucedió con nuestro paciente. Cabe recordar que la falta de respuesta al tratamiento antibiótico luego de un mes de instaurado, nos obliga a realizar algún procedimiento quirúrgico de drenaje de abscesos; además, se ha observado una mejor evolución clínica en los casos de actinomicosis torácica complicada (presencia de hemoptisis o fistula broncopleural) cuando se brinda tratamiento antibiótico y quirúrgico ${ }^{(1-3)}$.

La información sobre la actinomicosis diseminada cutánea es muy escasa. La inflamación cutánea progresiva y el desarrollo de abscesos o masas frías, asociados a disrupción de la membrana mucosa en cualquier parte del tracto gastrointestinal, torácico o pélvico, nos debe hacer sospechar en esta enfermedad ${ }^{(13)}$.

En conclusión, la actinomicosis diseminada con compromiso predominante de piel y tejidos blandos, sin daño pulmonar previo y en un paciente inmunocompetente es muy rara, y debe sospecharse ante la presencia de abscesos o masas frías, inclusive cuando estas tienen distribución difusa. Por otro lado, existe la posibilidad de desarrollar cuadro severo de actinomicosis a pesar de no contar con condiciones de inmunosupresión, siempre y cuando exista historia de disrupción de membrana mucosa. En relación al diagnóstico, es necesario realizar múltiples estudios. Finalmente, con relación al tratamiento, la actinomicosis diseminada responde bien a la terapia antibiótica con penicilina y, en pocos casos, es necesario la cirugía.

Contribuciones de los autores: ESM y JCS han participado en concepción y diseño del artículo, recolección y obtención de los datos, redacción y revisión crítica del artículo y en la aprobación de la versión final.

\section{REFERENCIAS BIBLIOGRÁFICAS}

1. Japanese Society of Chemotherapy Committee on guidelines for treatment of anaerobic infections, Japanese Association for Anaerobic Infection Research. Chapter 2-12-1. Anaerobic infections (individual fields): actinomycosis. J Infect Chemother. 2011 Jul; 17(Supl1):119-20.

2. Boyanova L, Kolarov R, Mateva L, Markovska R, Mitov I. Actinomycosis: a frequently forgotten disease. Future Microbiol. 2015; 10(4): 613-28.

3. Valour F, Sénéchal A, Dupieux C, Karsenty J, Lustig S, Breton $\mathrm{P}$, et al. Actinomycosis: etiology, clinical features, diagnosis,

treatment, and management. Infect Drug Resist. 2014 Jul 5; 7: 183-97.

4. Bonnefond S, Catroux M, Melenotte C, Karkowski L, Rolland L, Trouillier S, et al. Clinical features of actinomycosis: A retrospective, multicenter study of 28 cases of miscellaneous presentations. Medicine (Baltimore). 2016 Jun; 95(24): e3923.

5. Heo SH, Shin SS, Kim JW, Lim HS, Seon HJ, Jung SI, et al. Imaging of actinomycosis in various organs: a comprehensive review. Radiographics. 2014 Jan-Feb; 34(1): 19-33.

6. Cardona-Ospina JA, Franco-Herrera D, Failoc-Rojas VE, Rodríguez-Morales AJ. Estimaciones de la incidencia de la actinomicosis en Colombia. Enferm Infecc Microbiol Clin. 2017 Jun-Jul; 35(6): 331-98.

7. Munive L, Ramos W, Mendívil R, Paira E, Mendiola G, Chávez G. Actinomicosis de pared abdominal: reporte de dos casos en un hospital general de la ciudad de Lima. Rev Perú Epidemiol. 2009 Ago; 13(2): 1-5.

8. Mounsey JPD. Complete occlusion of the superior vena cava with chronic mediastinitis in a case of generalized actinomycosisby. Thorax. 1947 Dec; 2(4): 203-5.

9. Butas CA, Read SE, Coleman RE, Abramovitch H. Disseminated actinomycosis. Can Med Assoc J. 1970 Nov 7; 103(10): 1069-71.

10. Savidge RS, Davies DM. Generalized Actinomycosis with Possible Cardiac Involvement. Br Med J. 1953 Jul 18 ;2 (4828): 136.

11. Smith DL, Lockwood WR. Disseminated actinomycosis. Chest. 1975 Feb; 67(2): 242-4.

12. Takeda H, Mitsuhashi $\mathrm{Y}$, Kondo S. Cutaneous disseminated actinomycosis in a patient with acute lymphocytic leukemia. J Dermatol. 1998 Jan; 25(1): 37-40.

13. Bisero ED, Luque GF, Rizzo CN, Zapata AE, Cuello MS. Tuberculosis y actinomicosis. Un caso pediátrico de comorbilidad. Arch Argent Pediatr. 2016; 114(4): e233-6.

Fuentes de financiamiento

Este artículo ha sido financiado por los autores.

\section{Conflictos de interés}

Los autores declaran no tener ningún conflicto de intereses

Correspondencia:

Erick Sandoval Mallma

Dirección: Calle Mesones Muro N.- 109, Urbanización Maranga, San Miguel. Lima, Perú.

Teléfono: +51962354445

Correo electrónico: esandovalma@gmail.com

Recibido: 30 de noviembre de 2018 .

Evaluado: 01 de abril de 2019. Aprobado: 03 de mayo de 2019.

(c) La revista. Publicado por Universidad de San Martín de Porres, Perú. (cc) By Licencia de Creative Commons Artículo en acceso abierto bajo términos de Licencia Creative Commons Atribución 4.0 Internacional. (http://creativecommons.org/licenses/by/4.0/)

\section{ORCID iDs}

Erick Sandoval Mallma José Caballero Silva https://orcid.org/0000-0001-9247-5182 https://orcid.org/0000-0003-1124-285X 